\title{
Comparison of retina specialist preferences regarding spectral-domain and swept-source optical coherence tomography angiography
}

This article was published in the following Dove Press journal:

Clinical Ophthalmology

15 May 2017

Number of times this article has been viewed

\author{
Grace L Su' \\ Douglas M Baughman² \\ Qinqin Zhang ${ }^{3}$ \\ Kasra Rezaei ${ }^{2}$ \\ Aaron Y Lee ${ }^{2}$ \\ Cecilia S Lee ${ }^{2}$ \\ 'Lewis Katz School of Medicine, \\ Temple University, Philadelphia, PA, \\ ${ }^{2}$ Department of Ophthalmology, \\ ${ }^{3}$ Department of Bioengineering, \\ University of Washington, Seattle, \\ WA, USA
}

Purpose: The aim of this study was to compare physician preferences regarding the commercially available spectral-domain (SD) optical coherence tomography angiography (OCTA) and swept-source (SS) OCTA prototype device.

Design: Comparative analysis of diagnostic instruments was performed.

Patients and methods: Subjects at the University of Washington Eye Institute and Harborview Medical Center were prospectively recruited and imaged with the Zeiss SD OCTA (HD-5000, Angioplex) and Zeiss SS OCTA (Plex Elite, Everest) devices on the same day. The study included 10 eyes from 10 subjects diagnosed with a retinal/choroidal disease. Deidentified images were compiled into a survey and sent to retina specialists in various countries. The survey presented masked SD and SS images of each eye for each retinal sublayer side by side. Respondents were asked about their image preference and impact on clinical management. A priori and post hoc preferences for SD vs SS were collected.

Results: Fifty-four retina specialists responded to the survey. Median years in practice was 3.00 (interquartile range [IQR] 1.50-17.00). At baseline, 23 (48\%) physicians owned an OCTA machine. The majority of physician responses showed a preference for the SS over SD OCTA, independent of the retinal pathology shown ( $\mathrm{n}=454$ overall responses, $74 \%$ ). Nevertheless, the majority indicated that both SD and SS would be equally valuable in informing clinical decisions ( $\mathrm{n}=374$ overall responses, $61 \%$ ).

Conclusion: These findings indicate that the majority of retina specialists surveyed prefer SS over SD OCTA based on image quality, regardless of the retinal pathology shown. Regarding the clinical utility of each modality, the majority of physicians perceive SD and SS as equally effective.

Keywords: swept-source optical coherence tomography angiography, spectral-domain optical coherence tomography angiography, physician preference

\section{Background}

Healthy retinal and choroidal vasculatures are essential for the normal functioning of the eye. ${ }^{1}$ Abnormal growth of blood vessels as seen in choroidal neovascularization (CNV) can result from a variety of ophthalmologic diseases, such as neovascular agerelated macular degeneration (nAMD), high myopia, central serous chorioretinopathy (CSCR), and multifocal choroiditis. ${ }^{2}$ Quality retinal imaging techniques are critical in early detection and informing treatment decisions of these pathologies.

Optical coherence tomography angiography (OCTA) is a noninvasive imaging technique that utilizes the decorrelation motion contrast between sequential optical coherence tomography (OCT) B-scans to visualize retinal and choroidal blood flow
Correspondence: Cecilia S Lee Department of Ophthalmology, University of Washington, Box 359607 325 Ninth Avenue, Seattle,

WA 98104, USA

Tel +l 2068974809

Fax +I 2068974320

Email leecs2@uw.edu 
at a fixed point without the usage of contrasting agent. ${ }^{3}$ OCTA provides both structural (OCT) and functional (angiography) information, showing exact delineation and size measurements of flow, which allows for both to be evaluated in tandem. This novel technology generates volumetric angiography images within seconds and has the potential for detecting abnormalities in blood flow, giving it utility in identifying diseases such as age-related macular degeneration (AMD) and diabetic retinopathy. ${ }^{2}$

Currently available OCTA systems utilize the spectraldomain (SD) OCT software and operate at $\sim 840 \mathrm{~nm}$ wavelength. However, visualization beneath the retinal pigment epithelium (RPE) is partially obscured due to the backscattering of light from the RPE-Bruch's membrane complex. ${ }^{4,5}$ A newer OCTA prototype utilizes the faster swept-source (SS) OCT device that operates at a longer wavelength of $\sim 1,050 \mathrm{~nm}$, enabling enhanced light penetration into the deeper tissue. Other features of the SS OCT include a lower sensitivity roll-off, reduced fringe washout, and an ability to perform dual balanced detection, ${ }^{6}$ resulting in improved clarity and better visualization of the choroid on crosssectional and en face imaging. ${ }^{7}$

With growing evidence of the involvement of the choroid in retinal pathogenesis and the rise of OCTA as an imaging modality, it is important to evaluate the clinical utility of the SS and SD OCTA technologies. The purpose of our study is to compare SD (Zeiss US commercial) and SS (Zeiss research prototype) OCTA with the goal of assessing retina physicians' machine preferences and their effect on quality clinical management.

\section{Patients and methods}

This study was conducted at the University of Washington with surveys sent to outside retina specialists. The research adhered to the tenets of the Declaration of Helsinki and the Health Insurance Portability and Accountability Act. This study was approved by the University of Washington Institutional Review Board, and written informed consent was obtained prior to OCTA imaging.

\section{Subject selection}

Subjects seen by the retina service between June and September 2016 were recruited. Subjects were eligible if they had been previously diagnosed with a retinal pathology by a retina specialist, which was confirmed via a comprehensive chart review. Those with non-vascular retinal diseases such as retinal tears and lattice degeneration were excluded. The retinal/choroidal pathologies were categorized a priori as
1) retinal disease, which included diabetic retinopathy and branch retinal vein occlusion (RVO), 2) nAMD, and 3) RPE disease, which included CSCR, punctate inner choroidopathy, and multifocal choroiditis.

\section{Imaging acquisition}

We used a commercialized Zeiss SD OCTA (HD-5000, Angioplex) that operates at a central wavelength of $840 \mathrm{~nm}$ and an A-line speed of $68 \mathrm{kHz}$. The bandwidth of the light source is $45 \mathrm{~nm}$, contributing to an axial resolution of $\sim 5 \mu \mathrm{m}$ in the tissue and an estimated lateral resolution of $\sim 15 \mu \mathrm{m}$ at the retinal surface. We compared this with the Zeiss SS OCTA imaging system (Plex Elite, Everest), which utilizes a longer wavelength of $1,050 \mathrm{~nm}(1,000-1,100 \mathrm{~nm}$ full bandwidth) and operates at a faster speed of 100,000 A-lines per second. The axial and lateral resolutions are $\sim 5 \mu \mathrm{m}$ in the tissue and $\sim 14 \mu \mathrm{m}$ at the retinal surface.

All patients were imaged on the SD OCTA and SS OCTA within minutes of each other. All images were obtained as close to the fovea as possible. Since the 2 machines differ in the number of scan lines for a given retinal area, the SD and SS scans were taken over a $6 \times 6 \mathrm{~mm}$ and $9 \times 9 \mathrm{~mm}$ area, respectively, to assimilate the resolution in both machines. The subjects' fixation was controlled using the internal fixation target.

\section{Imaging analysis}

Ten eyes with high-quality signal strength and a diverse variety of retinal pathologies were selected. The sublayers of the retina as segmented by the manufacturer's default settings were shown, including the choriocapillaris, choroid, retina, superficial, deep, and avascular layers. The images were cropped and aligned to look visually equivalent but were otherwise unaltered. These scans were compiled into an online survey and sent to retina specialists in various countries, who were subsequently asked to send it to all of their colleagues. Surveys were initially sent to all retinal specialists for whom the authors could obtain an email address. Before completing the survey, retina specialists were informed via email of all pertinent information relative to study participation. Voluntary completion of the survey was considered consent to participate in all aspects of the study. The survey was designed as a web page opened within the users' web browser and submitted online. The machine from which the image was generated was not revealed to the respondents throughout the study. Each eye was presented as a case preceded by a clinical vignette and shown side by side with its corresponding layer (Figure 1). Each sublayer 

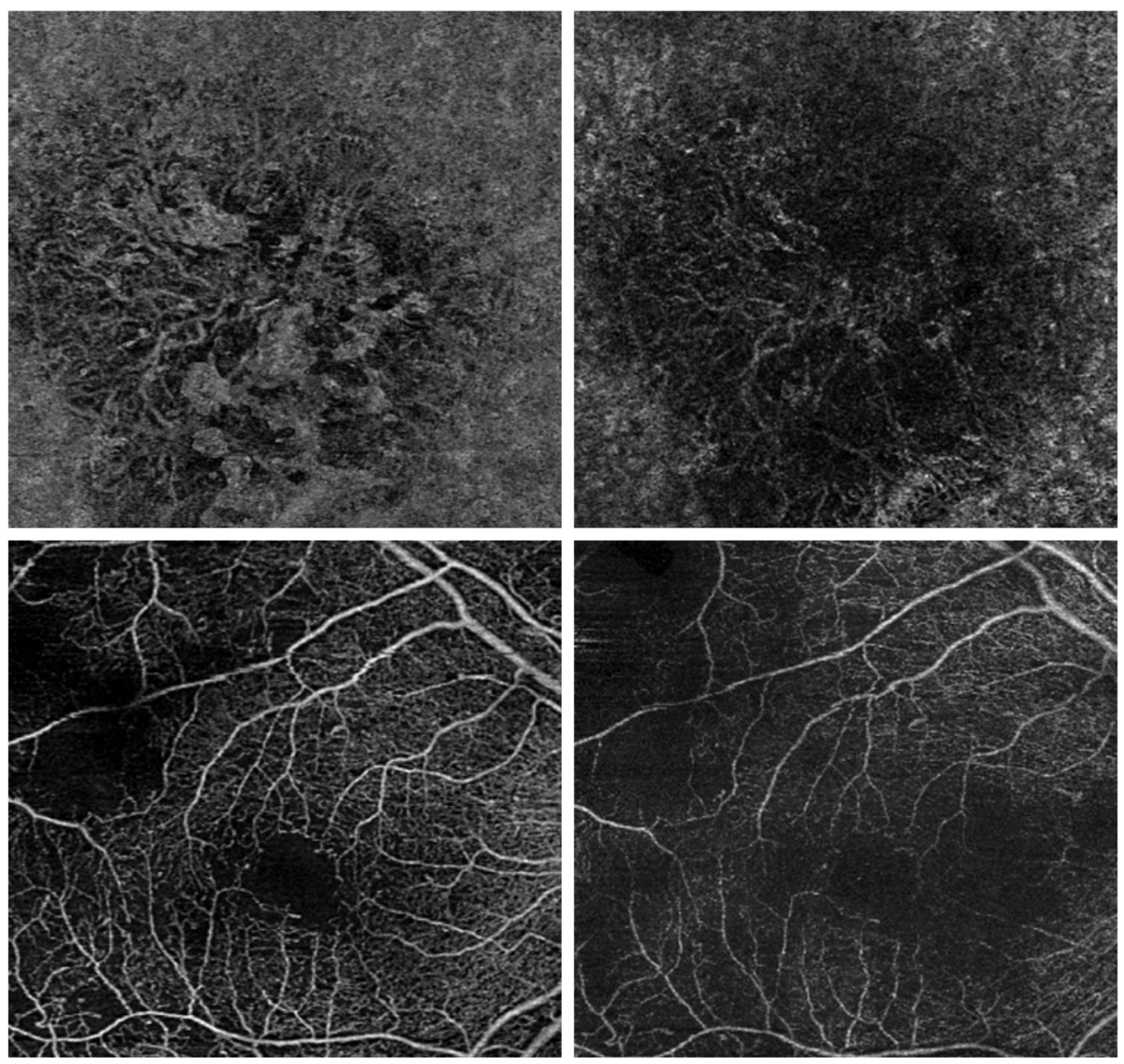

Figure I OCTA segmentation layers on Zeiss SS and SD.

Notes: Two cases shown as presented in the survey. AMD case showing the SS OCTA of the choriocapillaris (top left) and corresponding SD OCTA (top right). Diabetic retinopathy case showing the SS OCTA of the retinal layer (bottom left) and corresponding SD OCTA (bottom right).

Abbreviations: AMD, age-related macular degeneration; OCTA, optical coherence tomography angiography; SD, spectral domain; SS, swept source.

could be viewed by scrolling or clicking " $\mathrm{j}$ " or " $\mathrm{k}$ " on the computer keyboard. The order of the SD vs SS images for each case and the laterality of the images were randomized. Only one survey result per IP address was included for the analysis to prevent repeated submissions.

At the beginning of the survey, we collected demographic data including physicians' year of practice, type of practice, and practice location. We also asked about their OCTA purchase plan, OCTA preference, and opinions on clinical utility (Figure S1). Following each case, respondents were asked to select the image of higher quality in their assessment and which machine it came from. They were subsequently asked questions regarding clinical management preference (Figure S2).

\section{Results}

\section{Subject demographics}

Of the 15 image subjects selected, 13 completed the study, of which 10 subject eyes were selected. The remaining images were not used due to poorer imaging quality. One declined to participate due to language barriers, and another subject did not complete the study due to physical discomfort during imaging. The mean age of the study population was 59 (range $=24-84)$ years, and $5(50 \%)$ were male. Two subjects (20\%) had retinal diseases, 4 subjects ( $40 \%)$ had AMD, and 4 subjects (40\%) had RPE disease.

\section{Survey responses}

Surveys were emailed to retina specialists at multiple centers in various countries, including the USA, Europe, and Australia. Of the 54 retina specialists who filled out the survey, $32(67 \%)$ practiced in academic medical centers, $16(33 \%)$ in private practice, and 6 declined to answer. The physicians' median years in practice was 3.00 (interquartile range [IQR] 1.50-17.00). In response to the OCTA purchase plan question, 23 (48\%) already owned one, $8(17 \%)$ planned on obtaining one within a year, and $17(35 \%)$ were uncertain. In response to the question on the 
Table I Basic demographic characteristics and baseline OCTA attitudes of the survey respondents

\begin{tabular}{ll}
\hline Characteristics & Survey responses, $\mathbf{N}(\%), \mathbf{n}=\mathbf{5 4}$ \\
\hline $\begin{array}{l}\text { Years in practice, median (IQR) } \\
\text { Type of practice }\end{array}$ & $3.00(1.50-17.00)$ \\
Private practice & $16(33)$ \\
Academia & $32(67)$ \\
Missing & 6 \\
OCTA purchase plan & \\
Already own one & $23(48)$ \\
Within a year & $8(17)$ \\
Not sure & $17(35)$ \\
Missing & 6 \\
How useful is it clinically & \\
Definitely & $3(6)^{\mathrm{b}}$ \\
Sometimes & $17(35)^{\mathrm{b}}$ \\
Rarely & $13(27)^{\mathrm{b}}$ \\
Maybe in future & $15(31)^{\mathrm{b}}$ \\
Missing & 6 \\
OCTA preference & \\
SD & $12(28)$ \\
SS & $8(19)$ \\
SS if it was more affordable & $23(53)$ \\
Missing & 11 \\
\hline
\end{tabular}

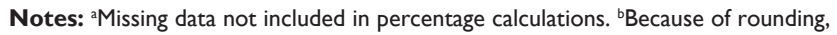
percentages may not total 100.

Abbreviations: IQR, interquartile range; OCTA, optical coherence tomography angiography; SD, spectral domain; SS, swept source.

effect of OCTA on clinical management, the answers varied from definitely needing an OCTA $(n=3,6 \%)$, to sometimes $(\mathrm{n}=17,35 \%)$, rarely $(\mathrm{n}=13,27 \%)$, and maybe in the future $(\mathrm{n}=15,31 \%)$. Prior to starting the survey, 23 (53\%) retina specialists preferred an SS OCTA over an SD OCTA device if it were more affordable (Table 1).

Based on the 617 overall responses, retina specialists were significantly ( $\mathrm{n}=454$ overall responses, $74 \%$ ) more likely to prefer SS OCTA over SD OCTA in terms of image quality (Table 2). However, this preference did not vary between retinal pathologies (retinal disease vs AMD vs RPE disease), and SS OCTA was predominantly preferred.

Regarding clinical management, the majority $(n=374$, $61 \%$ ) reported that both SD and SS would be equally valuable in making clinical decisions. This was seen consistently across all pathologies. A greater number of responses $(n=120$, $20 \%$ ) reported that only SS would be valuable, while a small minority $(\mathrm{n}=17,3 \%)$ thought that only SD would be useful. A total of $104(17 \%)$ overall responses reported that neither OCTAs would be useful in appropriate management (Table 2).

Prior to completing the survey, 12 of 43 (28\%) respondents indicated that they preferred the SD OCTA, $8(19 \%)$ preferred the SS, and $23(53 \%)$ preferred the SS if it were more affordable as their choice of imaging (Table 3). At the conclusion of the survey, 11 of 45 (24\%) respondents selected the SD OCTA, 13 (29\%) chose the SS, and 21 (47\%) chose the SS if it were more affordable. When analyzing changes in response upon termination of the survey, 5 of the $23(23 \%)$ respondents who originally preferred "SS OCTA if it were more affordable" changed their response to solely "SS OCTA" without knowing the correct answers to the survey. Of the 12 respondents who originally chose "SD OCTA," 8 (67\%) remained the same, $1(8 \%)$ changed their answer to "SS OCTA", and $3(25 \%)$ to "SS OCTA if were more affordable". With regard to the accuracy of matching the image to the machine, 262 of 430 times (61\%) the cases were correctly guessed.

\section{Discussion}

This study analyzed retina specialists' OCTA preferences when comparing SS to SD OCTA technologies. We surveyed

Table 2 OCTA preference pattern and clinical meaningfulness per various pathologies

\begin{tabular}{|c|c|c|c|c|}
\hline $\begin{array}{l}\text { Case } \\
\text { questionnaire }\end{array}$ & $\begin{array}{l}\text { Retinal disease, }{ }^{a} \\
N(\%), n=186\end{array}$ & $\begin{array}{l}\text { nAMD, N (\%), } \\
\text { n=247 }\end{array}$ & $\begin{array}{l}\text { RPE disease, } \\
N(\%), n=184\end{array}$ & $\begin{array}{l}\text { Overall, N (\%), } \\
n=617\end{array}$ \\
\hline \multicolumn{5}{|l|}{ Preference } \\
\hline SD & $37(20)$ & $67(27)$ & $55(30)$ & $159(26)$ \\
\hline SS & | 48 (80) & $179(73)$ & $127(70)$ & 454 (74) \\
\hline Missing ${ }^{c}$ & 1 & 1 & 2 & 4 \\
\hline \multicolumn{5}{|l|}{ Clinically valuable } \\
\hline SD only & $2(1)$ & $9(4)$ & $6(3)$ & $17(3)^{d}$ \\
\hline SS only & $40(22)$ & $55(22)$ & $25(14)$ & $120(20)^{\mathrm{d}}$ \\
\hline Both & $106(57)$ & $161(66)$ & $107(58)$ & $374(6 \mathrm{I})^{d}$ \\
\hline Neither & $38(20)$ & $20(8)$ & $46(25)$ & $104(17)^{d}$ \\
\hline Missingc & 0 & 2 & 0 & 2 \\
\hline
\end{tabular}

Notes: aRetinal disease includes diabetic retinopathy and branch RVO. ${ }^{\circ R P E}$ disease includes CSCR, punctate inner choroidopathy, and multifocal choroiditis. ${ }^{\mathrm{C}}$ Missing data

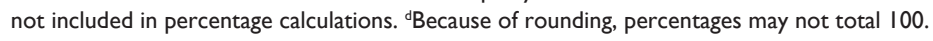

Abbreviations: CSCR, central serous chorioretinopathy; n, number of survey responses; nAMD, neovascular age-related macular degeneration; OCTA, optical coherence tomography angiography; RPE, retinal pigment epithelium; RVO, retinal vein occlusion; SD, spectral domain; SS, swept source. 
Table 3 OCTA preference pattern before and after the survey (without learning the correct answer during the survey)

\begin{tabular}{|c|c|c|c|c|c|}
\hline \multirow[t]{2}{*}{ Before survey } & \multicolumn{5}{|c|}{ After survey } \\
\hline & SD, N (\%) & SS, N (\%) & $\begin{array}{l}\text { SS if more } \\
\text { affordable, } \mathbf{N}(\%)\end{array}$ & Missing $^{a}$ & $\begin{array}{l}\text { Total, } \\
N(\%), n=43\end{array}$ \\
\hline SD & $8(67)$ & I (8) & $3(25)$ & 0 & $12(28)$ \\
\hline SS & $0(0)$ & $5(83)$ & I (17) & 2 & $8(19)$ \\
\hline SS if more affordable & $3(14)^{b}$ & $5(23)^{b}$ & $14(64)^{b}$ & I & $23(53)$ \\
\hline Missing ${ }^{\mathrm{a}}$ & 0 & 2 & 3 & 6 & \\
\hline Total, N (\%), n=45 & II (24) & $13(29)$ & $21(47)$ & & \\
\hline
\end{tabular}

Notes: aMissing data not included in percentage calculations. ${ }^{b}$ Because of rounding, percentages may not total 100.

Abbreviations: OCTA, optical coherence tomography angiography; SD, spectral domain; SS, swept source.

54 retina specialists to determine what, if any, preferences were apparent based on the 10 clinical cases given. With regard to image quality, the majority of retina specialists selected the SS over the SD OCTA. This preference did not vary between retinal pathologies. While our results did not reveal a significant partiality in either machine in terms of its effect on anticipated clinical management, we demonstrated that most clinicians preferred the SS OCTA as an imaging device.

Our analysis did not reveal OCTA in having a significant role in clinical management outside the currently available technologies, possibly due to its novelty and cost.

However, current literature highlights the potential of OCTA in becoming an important imaging modality in evaluating common ophthalmologic diseases, including diabetic retinopathy, ${ }^{8} \mathrm{AMD},{ }^{9,10}$ and RVO. ${ }^{11}$

Previous studies contrasting SS to SD prototypes have focused primarily on comparing physical parameters in specific diseases, such as measuring the choroidal thickness and detecting pathology. ${ }^{12}$ In a recent study, Novais et a ${ }^{12}$ compared the SS and SD OCTA technologies in their abilities to measure the total $\mathrm{CNV}$ area. They concluded that while both machines were able to identify feeder vessels, the SS OCTA yielded significantly larger CNV areas than did the SD OCTA in both type I and type II CNV, classified as when the neovascularization is below and above RPE, respectively. However, previous studies have not evaluated retina physicians' current preference patterns in OCTA machines, the primary goal of our study.

This is the first study to our knowledge that makes a comparison of preference between the SS and SD OCTA technologies. The study was blinded by keeping the machine prototypes anonymous to minimize viewer bias. In addition, we imaged subjects with various pathologies and surveyed retina specialists from a variety of practices across multiple countries. Retina specialists' preference of SS to SD OCTA did not vary across retinal or choroidal pathologies. This suggests that even the retinal diseases that had been previously imaged via SD, such as diabetic retinopathy ${ }^{8}$ and $\mathrm{RVO},{ }^{11}$ may show up more clearly via SS imaging. However, the analysis revealed that the majority found both machines to produce an adequate quality of imaging necessary to inform clinical management. We might have expected changes in management for the choroidal diseases, such as CNV secondary to AMD, as diseases beneath the RPE may be more easily visualized with SS imaging. Although there was no significant difference seen in anticipated clinical management, the improved delineation of the vasculature via the SS OCTA has the potential to advance our understanding of disease pathogenesis and consequently improve treatment.

Prior to starting the survey, about one-third of respondents indicated that they preferred the SD OCTA as an imaging technique. At the conclusion of the survey, there was a shift from the SD to solely the SS OCTA device irrespective of cost. This suggests an enhanced partiality for the SS images after repeated exposure, even with the randomization and anonymity of the machines. While the results indicated that neither machine had advantages over the other in terms its clinical utility, our analysis indicates a clear inclination for the SS OCTA device in terms of quality.

Our study has several limitations. Our statistical power was diminished due to a small sample size. Furthermore, the patients represented only a subset of retinal pathologies. Future studies comparing SS OCTA with SD OCTA across additional diseases will better demonstrate the capabilities of the newer technology. This includes pathologies such as Vogt-Koyanagi-Harada ${ }^{13}$ and pathologic myopia, ${ }^{14}$ which increase and decrease the choroidal volume, respectively. Additionally, we selected images with the best signal strength; the remainder were not used in this study. With regard to the survey, response bias may have been present due to already having seen the patient's diagnosis. Furthermore, those who owned an OCTA machine may have had an inherent preference compared to those who did not, which could have contributed additional bias. The median years in 
practice of survey respondents was also somewhat low, and our results may overrepresent the preferences of physicians in this demographic. Finally, we did not focus on other aspects of the imaging techniques, such as cost and machine handling, all of which influence a clinician's preference in managing patients.

This study compares the clinical utility of SS OCTA to the SD OCTA system. Although the SS OCTA enables better visualization of the choroidoscleral interface, the majority of retina physicians did not feel that the SS OCTA offers additional advantages over SD OCTA in our study cases. However, SS OCTA was overall preferred over SD OCTA due to the higher quality of images. Therefore, SS OCTA may become useful for finding subclinical manifestations of various subretinal diseases in the future. As OCTA availability continues to expand, its clinical utility in detecting and visualizing a greater number of diseases will be better understood. Future studies that include a larger sample are warranted to further investigate the clinical applicability of the SD and SS OCTA technologies with the goal of improving disease management.

\section{Acknowledgments}

The authors would like to acknowledge Thellea Leveque MD MPH at the Department of Ophthalmology, University of Washington, for her contribution with OCT imaging of a patient in the study. The content of this manuscript has not been previously presented at a meeting. This work was supported by the National Eye Institute, Bethesda, MD [K23EY02492] (CSL), the Research to Prevent Blindness, Inc., New York, NY (CSL, AYL, KR), and Carl Zeiss Meditec, Inc. The funding source had no role in the design and conduct of the study's collection, management, analysis, and interpretation of the data; preparation, review, or approval of the manuscript; and decision to submit the manuscript for publication.

\section{Author contributions}

CSL had full access to all the data in the study and takes responsibility for the integrity of the data and the accuracy of the data analysis. Study concept and design: AYL, CSL. Acquisition, analysis, or interpretation of data: AYL, CSL, GLS, QZ, KR. Drafting of the manuscript: GLS. Critical revision of the manuscript for important intellectual content: AYL,
CSL, KR, QZ, DMB. Statistical analysis: AYL, CSL, GSL. Obtaining funding: AYL, CSL, KR. Administrative, technical, or material support: AYL, KR, QZ. Study supervision: AYL, CSL. All authors contributed toward data analysis, drafting and critically revising the paper, and agree to be accountable for all aspects of the work.

\section{Disclosure}

The authors report no conflicts of interest in this work.

\section{References}

1. Huang Y, Zhang Q, Thorell MR, et al. Swept-source OCT angiography of the retinal vasculature using intensity differentiation-based optical microangiography algorithms. Ophthalmic Surg Lasers Imaging Retina. 2014;45(5):382-389.

2. de Carlo TE, Romano A, Waheed NK, Duker JS. A review of optical coherence tomography angiography (OCTA). Int J Retina Vitreous. 2015;1(1):5.

3. An L, Wang RK. In vivo volumetric imaging of vascular perfusion within human retina and choroids with optical micro-angiography. Opt Express. 2008;16(15):11438-11452.

4. Saito M, Iida T, Nagayama D. Cross-sectional and en face optical coherence tomographic features of polypoidal choroidal vasculopathy. Retina. 2008;28(3):459-464.

5. Manjunath V, Taha M, Fujimoto JG, Duker JS. Choroidal thickness in normal eyes measured using Cirrus HD optical coherence tomography. Am J Ophthalmol. 2010;150(3):325-329.

6. Drexler W, Liu M, Kumar A, Kamali T, Unterhuber A, Leitgeb RA. Optical coherence tomography today: speed, contrast, and multimodality. J Biomed Opt. 2014;19(7):071412.

7. Adhi M, Liu JJ, Qavi AH, et al. Choroidal analysis in healthy eyes using swept-source optical coherence tomography compared to spectral domain optical coherence tomography. Am J Ophthalmol. 2014;157(6): 1272-1281.

8. Ishibazawa A, Nagaoka T, Takahashi A, et al. Optical coherence tomography angiography in diabetic retinopathy: a prospective pilot study. Am J Ophthalmol. 2015;160(1):35-44.

9. Palejwala NV, Jia Y, Gao SS, et al. Detection of nonexudative choroidal neovascularization in age-related macular degeneration with optical coherence tomography angiography. Retina. 2015;35(11): 2204-2211.

10. El Ameen A, Cohen SY, Semoun O, et al. Type 2 neovascularization secondary to age-related macular degeneration imaged by optical coherence tomography angiography. Retina. 2015;35(11):2212-2218.

11. Kashani AH, Lee SY, Moshfeghi A, Durbin MK, Puliafito CA. Optical coherence tomography angiography of retinal venous occlusion. Retina. 2015;35(11):2323-2331.

12. Novais EA, Adhi M, Moult EM, et al. Choroidal neovascularization analyzed on ultrahigh-speed swept-source optical coherence tomography angiography compared to spectral-domain optical coherence tomography angiography. Am J Ophthalmol. 2016;164:80-88.

13. Rajendram R, Evans M, Rao NA. Vogt-Koyanagi-Harada disease. Int Ophthalmol Clin. 2005;45(2):115-134.

14. Quaranta M, Arnold J, Coscas G, et al. Indocyanine green angiographic features of pathologic myopia. Am J Ophthalmol. 1996;122(5): 663-671. 


\title{
Supplementary materials
}

\author{
OCTA SD vs SS Survey \\ Purpose Info Case 1 Case 2 Case 3 Case 4 Case 5 Case 6 Case 7 Case 8 Case 9 Case 10 Final \\ Years since fellowship: 0 if still in training, 1 if first year after fellowship, etc. \\ Type of practice: $\bigcirc$ Academic $\bigcirc$ Private \\ Plan to obtain OCTA machine: $\bigcirc$ Already have one $\bigcirc$ Planning to get one within the year \\ Not sure if one is necessary $\bigcirc$ Never planning to get one \\ In your opinion, how often do you think $\bigcirc$ Definitely $\bigcirc$ Sometimes $\bigcirc$ Rarely $\bigcirc$ Not yet, but may in the future \\ OCTA affects your clinical management? \\ If you have to buy one OCTA which would it be? $\bigcirc$ SD OCTA $\bigcirc$ SS OCTA $\bigcirc$ SS OCTA if it were more affordable \\ Previous \\ Next
}

Figure SI Screenshot of questionnaire at the beginning of the survey.

Note: Created by Aaron Y Lee and Cecilia S Lee, University of Washington.

Abbreviations: OCTA, optical coherence tomography angiography; SD, spectral domain; SS, swept source.

In your opinion, which is the higher quality OCTA $\bigcirc \mathrm{A} \bigcirc \mathrm{B}$

image:

Which machine do you think the image you picked $\bigcirc$ Cannot decide $\bigcirc$ Zeiss SD OCTA $\bigcirc$ Zeiss SS OCTA came from?

Which OCTAs are good enough for appropriate $\bigcirc$ Only the better image $\bigcirc$ Both choices $\bigcirc$ Neither would be useful management?

Figure S2 Screenshot of questionnaire following each case.

Note: Created by Aaron Y Lee and Cecilia S Lee, University of Washington.

Abbreviations: OCTA, optical coherence tomography angiography; SD, spectral domain; SS, swept source.

Clinical Ophthalmology

\section{Publish your work in this journal}

Clinical Ophthalmology is an international, peer-reviewed journal covering all subspecialties within ophthalmology. Key topics include: Optometry; Visual science; Pharmacology and drug therapy in eye diseases; Basic Sciences; Primary and Secondary eye care; Patien Safety and Quality of Care Improvements. This journal is indexed on

Submit your manuscript here: http://www.dovepress.com/clinical-ophthalmology-journal
Dovepress

PubMed Central and CAS, and is the official journal of The Society of Clinical Ophthalmology (SCO). The manuscript management system is completely online and includes a very quick and fair peer-review system, which is all easy to use. Visit http://www.dovepress.com/ testimonials.php to read real quotes from published authors. 